\title{
Lumen
}

Selected Proceedings from the Canadian Society for Eighteenth-Century Studies

\section{Les notions de machine et de mécanisme : conditions et possibilités des métaphores de la machine dans la pensée de l'âge classique}

\section{Jean-Luc Martine}

Volume 25, 2006

URI : https://id.erudit.org/iderudit/1012073ar

DOI : https://doi.org/10.7202/1012073ar

Aller au sommaire du numéro

Éditeur(s)

Canadian Society for Eighteenth-Century Studies / Société canadienne d'étude du dix-huitième siècle

ISSN

1209-3696 (imprimé)

1927-8284 (numérique)

Découvrir la revue

Citer cet article

Martine, J.-L. (2006). Les notions de machine et de mécanisme : conditions et possibilités des métaphores de la machine dans la pensée de l'âge classique. Lumen, 25, 1-16. https://doi.org/10.7202/1012073ar

Copyright (c) Canadian Society for Eighteenth-Century Studies / Sociéte canadienne d'étude du dix-huitième siècle, 2006
Ce document est protégé par la loi sur le droit d'auteur. L'utilisation des services d'Érudit (y compris la reproduction) est assujettie à sa politique d'utilisation que vous pouvez consulter en ligne.

https://apropos.erudit.org/fr/usagers/politique-dutilisation/ 


\section{Les notions de machine et de mécanisme : conditions et possibilités des métaphores de la machine dans la pensée de l'âge classique}

L'âge classique accorde une large importance à l'idée de la machine. La notion investit l'ensemble de l'encyclopédie des savoirs, où l'on constate son empreinte aussi bien dans les champs constitués que dans ceux qui connaissent des déplacements et qui sont en cours de reconstitution (techniques, sciences, philosophie, pensée politique, littérature, arts, esthétique). Cette emprise à été étudiée par secteur, mais elle ne l'a pas été dans son ensemble. La sectorisation, qui résulte du succès de l'idée, constitue l'une des difficultés que l'on doit affronter. Mais ce n'est pas la seule. Il est en effet nécessaire de comprendre le rôle qu'a pu jouer l'idée de machine en amont du romantisme et de la "révolution machiniste», où s'élabore une forme d'opposition entre "machine» et «organisme» dont nos représentations modernes sont largement tributaires. Comme le montre Judith Schlanger ${ }^{1}$, le romantisme a imposé des conceptions de la machine qui servent de contre-modèle destiné à justifier la tournure nettement vitaliste de sa vision du monde et de sa perception de l'idée de totalité. Or, cette dévalorisation de la machine ne correspond pas aux intuitions de l'âge classique. Il peut alors s'agir d'entreprendre une archéologie et une généalogie de la structure de l'idée classique de machine et des métaphores qu'elle rend possibles, qui nous renseignent sur les catégories reçues aussi bien que sur les déplacements et les redistribu-

1 Judith Schlanger, Les Métaphores de l'organisme, Paris, Vrin, 1971 ; réédition : Paris, L'Harmattan, 1995. 
tions opérées par certaines des tendances et des forces qui parcourent les $\mathrm{XVII}^{\mathrm{e}}$ et XVIII ${ }^{\mathrm{e}}$ siècles. Ce projet nous place cependant dans la situation particulièrement difficile d'avoir affaire à une profusion de discours hétérogènes, à un ensemble de textes très disparates qui dessinent une nébuleuse verbale dont la machine, si elle en est le noyau, ne l'est que rarement comme l'objet même du discours qui la mobilise. A la conscience de l'importance évidente de quelque chose, au cœur de la pensée classique, qui se signale par l'utilisation, au niveau le plus élémentaire, du mot «machine» s'ajoute encore une forme particulière $d^{\prime}$ inconfort : celle de machines "insolubles», apparaissant dans des énoncés dont il faut reconnaître l'étrangeté (particulièrement chez Diderot). Il semblerait enfin qu'il faille tenir compte de l'association de la machine avec le mécanisme, qui paraissait s'imposer comme une ligne d'interprétation déjà constituée. Or, le caractère univoque de la rationalisation cartésienne de l'idée de machine est une illusion dont il importe de se déprendre.

L'évidente multiplicité de l'idée de machine s'oppose à toute tentative de repliement sur un concept transparent. Pour reconstituer les tensions qui rendent la notion à la fois riche et instable il faut construire artisanalement les procédures capables de rendre compte des plans où s'étagent les composantes sémantiques impliquées dans le lexique de la machine. C'est de là que procède la nécessité de recourir aux dictionnaires. Il apparaît en effet rapidement que le discours analogique reste largement incompréhensible lorsque l'on ne dispose pas du répertoire des valeurs signifiantes attachées aux termes que le français a choisi pour signifier les idées sur lesquelles les métaphores de la machine peuvent s'articuler. Avant d'être en mesure de statuer sur le discours des métaphores, il convient de comprendre ce que signifie «à proprement parler» la notion de machine et quelles sont les valeurs que l'âge classique a pu conférer aux termes de l'univers de la machine. Cela permet d'établir les conditions de possibilité de l'étude des métaphores machiniques. Le discours des dictionnaires ${ }^{2}$ révèle aussi bien la singulière étendue de l'idée de machine (qui s'applique à des référents aussi peu mécaniques qu'un enduit de cordonnier, une échelle, un clapier ou des castagnettes) que les harmoniques sémantiques qui l'associent continûment au secret, à la dissimulation des causes et des

2 Ce discours a été lu de la naissance de la lexicographie française (dans les ouvrages issus du travail de Robert Estienne, publiés entre 1530 et 1606) jusqu'à la cinquième édition du Dictionnaire de l'Académie (1798), en passant par ce Dictionnaire raisonné qu'est aussi l'Encyclopédie. 
procédures, aux mystères de l'invention créatrice et, en général, à toutes les formes de l'inconnu ou du méconnu. Il devient possible de préciser la disposition d'un ensemble de tensions et de contraintes, à partir d'une dynamique et de ses lignes de force, ce qui permet de penser l'histoire du lexique en rapport avec une archéologie, en tentant de neutraliser les deux notions associées à celle d'archéologie : la recherche de l'arché et l'idée de fouille. Il importe donc d'adopter une position qui isole les types de discours où «machine» intervient afin d'en faire une description qui soit résolument une description de surface, passant par des relevés, des descriptions de familles, des délimitations de frontières mobiles. A partir de ces descriptions, nous proposons une méthode, dont l'esprit emprunte sa tournure à la manière dont Nietzsche envisage la notion de généalogie, afin de se tenir le plus éloigné possible de toute recherche d'un archétype. Il ne s'agit pas d'envisager le lexique du point de vue de sa réception, mais du point de vue de sa création, en déterminant les intentions, les forces, les puissances, les capacités d'action qui ont pu déterminer l'invention d'un mot ou l'investissement d'un vocable par une idée neuve. Le discours lexicographique se présente alors comme un répertoire de conflits entre forces antagonistes. Les dictionnaires sont des témoins : les acceptions s'y déposent en laissant la trace des intentions qui s'emparent successivement des mots ou qui s'y formulent simultanément et de manière concurrente. Cette lecture se justifie d'autant mieux que ce qui s'exprime dans le lexique de la machine, ce sont des relations de puissance (au sens de capacité d'agir) : puissance créatrice de la pensée inventive, puissance technique du machiniste ou du mécanicien, puissance analytique de connaître, puissance économique de produire, puissance spectaculaire d'étonner ou de produire un effet puissant. L'histoire de l'idée de machine forme ainsi un petit canton pas forcément tranquille de celle des idées. Elle permet d'envisager certains aspects de la rationalité occidentale où l'on peut suivre la ligne, dissimulée mais durable, que trace la forme particulière et énigmatique de fécondité de l'esprit qui a trouvé dans le lexique de la machine une expression privilégiée, capable d'en faire un espace où la littérature (au sens encyclopédique et esthétique) tente d'organiser sa résistance à la géométrisation du monde.

L'orientation lexicale et sémantique de notre enquête rend nécessaire l'étude de la littérature gréco-latine, qui est la source à laquelle le français emprunte les mots qui lui servent à penser la machine, mais qui se présente surtout comme un inépuisable répertoire d'images et de comparaisons où l'âge classique invente ses propres ressources expressives. Le vocabulaire grec de la mèkhanè permet de faire apparaître la relation profonde entre certains traits de la machine et la figure de Métis, à laquelle Marcel Détienne et Jean-Pierre Vernant ont consacré une étude 
décisive ${ }^{3}$. Le français «machine» est en effet une transposition érudite du latin machina, qui appartient à l'ensemble des termes formés à partir d'un emprunt au dorien machana, lui-même issu de l'ancien radical *magh-, dont le sens s'attache à la notion de «pouvoir», où il faut entendre l'expression d'une puissance comprise comme une capacité d'agir. Les significations concrètes apparaissent avec mèkhanè pour désigner ce que nous nommons "machines» (les machines de guerre et machineries théâtrales), mais le sens premier et dominant du terme est celui de "moyen», mèkhanè désignant «toute espèce de moyens, de combinaisons, $\mathrm{d}^{\prime}$ inventions ${ }^{4}$. Le noyau sémantique de mèkhanè et de tous les mots grecs de sa famille est ainsi constitué par la référence à la forme de capacité qui résulte de l'invention ingénieuse de moyens et d'expédients. Les mots formés sur cette racine ancienne désignent la forme particulière d'intelligence qui préside aussi bien à la mise en œuvre de conduites efficaces qu'à la conception d'agencements matériels. Ce qui est perçu dans les objets, c'est l'esprit dont ils procèdent : une intelligence pratique pleine de ressources, voire une connivence secrète avec quelque force occulte. Ce trait d'ingéniosité constitue la première puissance qui, dans la variation complexe des significations dont nous proposons la généalogie, trouve son expression dans le vocabulaire de la «machine». Parmi les éléments qui s'expriment dans ce lexique, c'est peut-être aussi avec la puissance d'agir issue de cette mystérieuse capacité d'inventer des retournements ingénieux que l'idée de machine conservera le plus d'affinités.

Par ailleurs, une juste appréciation des composantes sémantiques et de leur environnement intellectuel peut permettre d'éviter de céder aux tentations de l'interprétation rétrospective. L'attention portée au contexte permet, par exemple, d'interpréter les analogies qu'Aristote propose entre les formes naturelles et la sphère technique en des termes qui ne permettent aucunement de penser l'identification de l'art et de la nature. La rencontre entre l'art et la nature prend un tour particulièrement frappant dans l'œuvre de Galien, mais l'agencement des organesinstruments ne possède pas le caractère mécaniste qui permettrait de faire de sa biologie une technologie puisque l'unité et la perfection des assemblages reposent sur l'idée stoïcienne de sympathie qui garantit

3 M. Détienne et J.-P. Vernant, Les Ruses et l'intelligence. La Mètis des grecs, Paris, Flammarion, 1974. Nos remarques lexicales montrent tout ce que nous devons à cette étude.

4 Pierre Chantraine, Dictionnaire étymologique de la langue grecque. Histoire des mots, Paris, Klincksieck, 1968, p. 699-700. 
seule la cohésion du Tout. L'image de l'automate, lorsqu'elle intervient, ne développe pas un imaginaire de la communication des mouvements, mais repose sur l'efficience d'une législation préalable qui évite l'intervention constante du Créateur dans sa création. La notion de sympathie n'est pas mécaniste : elle renvoie à une origine, non à une relation actuelle entre les composantes d'une totalité harmonieuse. Ainsi, la biologie permet de montrer l'importance de cette machine non mécaniste dont l'influence sera grande dans les siècles ultérieurs et dont le cartésianisme devra se débarrasser, ce qu'il ne pourra jamais faire complètement. La biologie laisse alors surtout la place à des métaphores qui renvoient à l'astuce du Créateur, à son habileté bienveillante investie dans l'art de dispositions qui ne répondent pas à l'idée que se formera la pensée mécaniste de la structure d'une machine. De la même manière, la cosmologie fortement artificialiste de Platon propose une figure, celle du démiurge, qui fait porter l'accent sur le moment intellectuel et prémédité du dessein créateur, non sur les structures formées.

La structure du vocabulaire latin conditionne largement l'histoire du lexique français de la machine. Le substantif machina est un emprunt pré-littéraire au dorien machana (le latin ne reproduisant pas l'attique mèkhanè, sauf dans quelques emprunts tardifs et, dans l'ensemble, peu vivants). Cette «anomalie» ouvre la possibilité d'un dédoublement qui détermine l'histoire du sémantisme et des idées : elle rend disponible le vocabulaire grec pour toute une série de réemprunts qui seront investis par des représentations intellectuelles largement divergentes de celles que lègue la configuration latine. Ainsi, la structure du lexique traduit, sous la forme d'une bipartition entre le vocabulaire du mécanisme et celui de la machine, un ensemble de tensions intellectuelles dont se nourrira la pensée de l'âge classique. Le déplacement sémantique qui s'opère dans le passage de mèkhanè à machina repose sur un changement de perspective : mèkhanè nommait une forme d'intelligence dont on reconnaissait les procédures typiques dans le fonctionnement particulier de certains objets fabriqués ; machina désigne une catégorie de moyens matériels dont la mise en œuvre peut requérir une forme de compétence ingénieuse, essentiellement pratique. Mais, en même temps que le latin se dote avec machina d'un générique capable de désigner une catégorie d'objets fabriqués, la référence à l'ingéniosité caractéristique de la mètis ne s'efface pas, puisque la plupart des mots de la famille portent ce trait sémantique. La permanence des valeurs grecques dans le vocabulaire latin est déjà un phénomène d'influence et de cumul, dont la possibilité est garantie par les échanges entre deux systèmes linguistiques coprésents : pour les Latins le grec est une langue vivante à partir de laquelle peuvent être directement adaptées la plupart des significations «morales» (où machina prend directement le sens de moyen ingénieux et 
de stratagème, sans passer forcément par la référence indirecte à un objet mécanique), lesquelles doivent leur étrangeté (leur impertinence relative interprétée en termes de «figure») à cette transposition ${ }^{5}$.

La langue latine déploie une ample configuration lexicale agencée autour de machina, dont dépend directement celle qui se formera et évoluera dans le patrimoine linguistique français. Le Thesaurus linguae latinae ${ }^{6}$ (héritier des ouvrages dont la Renaissance a établi le type) nous a permis de nous orienter dans l'exploration des significations de machina et de celles des mots de sa famille, qui composent, dans l'ensemble de la latinité, un véritable feuilleté de strates sémantiques dont il importait d'explorer la sédimentation cumulative. La configuration latine conditionne en effet l'évolution du vocabulaire de la machine. Mais cet héritage terminologique n'est qu'une part de celui que la République des Lettres reçoit d'une latinité qui se dispose aussi comme un immense répertoire de concepts et d'expressions, très tôt commentés et repris, où la pensée classique continue de puiser une part importante de ses ressources expressives. L'ensemble des éléments qu'il comporte définition, typologie, relation avec les notions voisines, lien au thème de la machine du monde, influence sur les représentations ultérieures permet d'utiliser le De architectura de Vitruve comme le point à partir duquel organiser la description du lexique de la machina. La définition que propose Vitruve de la machina est par ailleurs remarquable par sa portée réduite et défectueuse en regard de ce qui se trouve, dans le texte même du traité, nommé machina. Le point de vue choisi par Vitruve ne lui permet pas d'unifier les significations du terme qu'il entend définir. Machina peut désigner un simple assemblage, une construction sans parties mobiles (un échafaudage) mais aussi des procédés qui ne sont plus du tout des choses et qui participent encore de la mètis grecque (les

5 De même, le français continuera longtemps d'emprunter du latin des signifiants auxquels les emplois plus modernes ne feront que s'ajouter, dans un processus amplement cumulatif où les valeurs «anciennes» ne s'effacent pas toujours au profit des plus «modernes». C'est ce phénomène d'emprunt, qui crée très littéralement une étrangeté dans la langue, que les dictionnaires classiques traduisent en termes de figure, ce d'autant plus volontiers que la langue tente souvent de réinvestir le sémantisme que «machine» reçoit de mèkhanè via machina par des valeurs contemporaines, pour créer des expressions comme «les ressorts secrets de cette machine /machination".

6 Thesaurus linguae latinae, editus iussis et auctoritate consilli ab academiis societatibusque diversarum nationum electi, Volumen VIII M, MCMXXXVI-MCMLXVI, Lipsiae in aedibus, Stuttgart et Leipzig, B G teubner. Les articles sur les termes qui nous occupent se trouvent aux p. 11-18 et 515-516. 
stratagèmes). La définition qu'il propose oscille entre des extrêmes qui lui échappent : la construction pratique (le fait même de construire les machines), la science des mouvements (la mécanique grecque, celle du Pseudo-Aristote ou d'Archimède) et l'ingéniosité fertile en expédients (la source originelle: Mètis). Le texte de Vitruve témoigne indirectement du double conflit qui s'instaure, au sein du domaine circonscrit par l'idée de machine, entre les diverses sortes de relations que peuvent entretenir l'esprit et la matière (la compréhension des principes s'opposant à la construction d'agencements techniques) et les formes d'intelligence qui se pressent autour de la machina (ingéniosité et raison connaissante). La machina (puis la machine) hésite ainsi entre les trois formes de pouvoir qu'elle représente et qui l'investissent tour à tour : les ressources de l'esprit qui se joue des fluences du monde, l'artifice constructiviste et technique et la puissance de la raison. Le symptôme de ce conflit, c'est l'incapacité de produire une définition capable de rendre compte de l'ensemble de ce que le traité nomme machina.

Pour déplier le sens de machina et déterminer quelles sont les puissances qui investissent la notion et qui s'expriment en elle, on peut alors suivre les indications déposées dans la mémoire de la langue. C'est dans cet esprit qu'ont été étudiées la plus grande partie des occurrences de machina et des mots de sa famille ainsi que les analogies auxquelles ces termes ont pu donner naissance. Le premier type de contexte où machina inscrit l'idée de puissance qui s'attache à son sémantisme concerne le domaine militaire : machina intervient toujours dans le cadre de la guerre de siège où il s'agit, pour remporter la victoire, de faire appel aux ressources inventives et aux compétences techniques de l'architectus afin de résoudre les difficultés pratiques posées par l'art des fortifications. Machina correspond alors à un ensemble très disparate de constructions - elles vont des échelles, baraques et tours roulantes, aux balistes, catapultes et scorpions - auxquelles les lexicographes de l'âge classique accorderont toujours une place considérable. La machina relève nettement de l'objet fabriqué, mais s'incarne en elle l'ingéniosité mercenaire capable de concevoir et de mettre en œuvre ces diverses constructions, dont seules les machines de jet comportent quelque chose comme un moteur, produisant un mouvement puissant qui surpasse les forces humaines. L'une des caractéristiques importantes de ces machinae est de conserver une certaine mobilité. Leur conception doit être en effet assez souple pour s'adapter aux différents terrains d'opération où elles peuvent entrer en action. C'est cette nécessaire adaptation, où tout est sans cesse à repenser, qui empêche leur identification aux procédures routinières des métiers et à l'universalité de la science.

Les images impliquant la référence à la machina reposent sur les notions (refusées ou revendiquées) de puissance et/ou de moyen 
ingénieux. Elles ne laissent entendre aucune représentation structurelle, et elles sont généralement indifférentes à ce que sont ces machinae, comme dans les expressions déjà convenues que le latin emprunte au grec, où les machines de guerre illustrent les ressources de l'esprit. Le seul motif de ces analogies réside dans le caractère d'expédient rusé intervenant face à une puissance adverse. Si le français classique fait un ample usage de ces mêmes images, il ne fait pas intervenir une compréhension plus nette de la structure des objets qui servent à décrire les formes d'action désignées comme des «machines». La valeur expressive $\mathrm{du}$ terme, en français comme en latin, s'accommode fort bien d'une certaine ignorance. Le lexique de la machina entretient par ailleurs des liens privilégiés avec certains types de discours, ce qui aura des conséquences importantes pour les emplois de «machine» à l'âge classique, où se développe toute une théorie des registres. Machina est associé au style élevé de l'épopée, de l'histoire (pour les valeurs matérielles) et de la rhétorique (pour les emplois «figurés»), ou bien au style bas du comique (pour certains des expédients rusés).

Dans le domaine civil, et non plus militaire, quatre traditions sont en concurrence : celle des thaumata (automates et merveilles mécaniques), celle des mécaniques (les puissances paradoxales des machines simples), celle des moyens de production (tours et meules) et celle des structures provisoires (gradins, estrades, échafaudages et chevalets), parmi lesquelles la machine de levage opère une sorte de synthèse. Machina s'applique à des constructions dont les caractéristiques techniques sont, là aussi, très disparates. L'association de machina à l'ordre de la production et de la fabrication ou bien à celui des merveilles inutiles ne lui permet pas de recevoir une valorisation équivalente à celle acquise dans le registre militaire. Dans ce contexte civil, les rares énoncés analogiques restent dans l'esprit des Questions mécaniques, et font intervenir l'idée d'une puissance paradoxale où le petit agit sur le grand. Dans un registre «biologique», diverses machinae ont pu intervenir, à titre d'analogies, au sein de pensées artificialistes ou providentialistes, qui relèvent de deux traditions : celle du finalisme stoïcien, fondée sur l'idée d'une Natura providentielle et bienveillante, et celle qui procède de l'artificialisme platonicien. Lorsque s'agit de penser les relations entre le tout et ses parties, le vocabulaire de la machina continue de renvoyer à l'idée d'une convenance harmonieuse illustrant un dessein providentiel. Il ne s'agit jamais de l'application d'un modèle mécaniste permettant de comprendre une totalité complexe. Lorsque l'homme lui-même devient machina, dans des énoncés où la théologie chrétienne récupère le providentialisme ancien, le terme décrit son caractère de créature où s'unissent un corps façonné et une âme. Machina désigne l'homme dans sa relation privilégiée au créateur, ce qui permet de mesurer quel travail il faudra 
opérer sur l'idée de machine pour que la proposition d'un «homme machine» puisse devenir dangereusement matérialiste.

D'une manière générale, la machina permet le déploiement d'un lien en amont avec l'intelligence dont elle procède (dont les agencements fonctionnels sont la preuve). Elle est toujours l'occasion, dans les contextes philosophiques où elle intervient, de remonter vers un esprit, une intelligence ouvrière avisée. Comprendre une machine c'est y lire un dessein, une intention. Ce qu'elle permet de percevoir dans la nature, ce sont des fins. Elle n'est pas en elle-même un modèle pour une connaissance qui chercherait à établir sa juridiction et ses pouvoirs en dehors du règne des intentions. Ce qu'inventera la science moderne, et qui s'appellera mécanisme, c'est précisément un retrait de l'esprit, un repli des intentions : connaître ne sera plus concevoir un dessein dont les agencements seront des indices, mais concevoir par quels mécanismes sont produits les phénomènes.

Le Moyen Âge, s'il n'a pas fait grand usage du mot «machine», n'a ignoré ni le lexique latin issu de machina, ni certains des agencements techniques qui peuvent correspondre à l'idée de machine. Il se trouve que le français médiéval ne nomme pas, sauf exception, les objets susceptibles de répondre à l'idée de machine par des termes qui seraient issus de machina. Il a eu recours pour cela à un autre lexique, formé à partir d'ingenium. C'est en effet le terme «engin» qui, au Moyen Âge, se voit chargé, avec un ensemble de significations morales qui diffèrent sensiblement de celles qui étaient associées au lexique latin de la machina, des valeurs matérielles qui correspondent au sens concret de «machine». Le premier lexique français formé sur machina désigne l'exercice d'une faculté. $\mathrm{Si}$ «machiner», en moyen français, peut avoir la signification neutre de «supposer, envisager», «machineur» se rapporte au complot, à la trame secrète de desseins illégitimes. Sur l'autre versant de la configuration, les termes français construits sur mechanicus, et contemporains du développement d'emprunts latins tardifs au lexique grec de la mèkhanè, n'ont aucun lien avec l'idée de machine : ils signifient constamment l'évaluation d'une qualité. Ils renvoient alors à la catégorie dépréciée de l'artisanat et des pratiques «manuelles», associées à l'idée de servitude.

Les éléments que procure la lexicographie médiévale permettent par ailleurs d'envisager quelques-unes des évolutions significatives de l'idée de machina dans le Moyen Âge latin. La plus importante concerne le lien qui s'établit par le biais de la tromperie entre machina et la nature désormais pécheresse de l'esprit humain. La machine, c'est la pente du diable et de l'hérésie. Les significations concrètes, si elles conservent leurs acceptions antiques, tendent cependant à évoluer vers la désignation de constructions et d'édifices qui n'ont plus le caractère de moyen 
qu'enveloppe la terminologie latine. C'est, enfin et surtout, dans le latin médiéval que se développent les analogies qui permettent à machina de s'appliquer au monde ou à l'homme, en tant qu'ils sont des créatures. Le vocabulaire de la machina participe de l'expression d'un univers (et de ses créatures) conçu(es) comme le produit d'un agencement, qui trouve en partie ses cautions dans le domaine de l'architecture, mais aussi d'autres arts, selon une perspective qui semble faire la synthèse métaphorique de l'artificialisme platonicien et de la notion stoïcienne de Providence, ce qui invite à retracer les éléments lexicaux qui participent de la machina mundi depuis Lucrèce jusqu'à Lactance. Ces développements permettent de comprendre le contexte dans lequel le mot «machine» fait sa première entrée en français par transposition du latin, là où Oresme, traduisant et commentant Aristote, ne trouve pas dans le lexique français de l'engin les valeurs cosmologiques que la théologie et l'ésotérisme médiévaux ont attaché à la machina mundi.

La lexicographie du $\mathrm{XVI}^{\mathrm{e}}$ siècle permet de comprendre comment la restitution d'une latinité comprise comme «classique» contribue à obscurcir le sens de cette machina médiévale, encore largement présente dans le Dictionarium de Calepino, mais qui disparaît tout à fait du Thesaurus de Robert Estienne. Cet obscurcissement par scrupule philologique permet à «machine» de faire une seconde entrée en français, dans le contexte de l'archéologie livresque au moyen de laquelle la lexicographie renaissante tente, à partir de sources difficiles à interpréter et souvent contradictoires, la reconstitution des référents latins et grecs. Mais cette «relatinisation» a aussi pour conséquence de libérer machina de certaines de ses attaches signifiantes et d'en faire un vocable à la fois peu déterminé et fortement valorisé où pourra s'inscrire un vaste procès $d^{\prime}$ invention métaphorique. C'est néanmoins encore l'âge d'une machina érudite, dont les éléments influent sur les déterminations d'un vocable qui tarde à s'implanter en français et à supplanter le lexique ancien, issu d'ingenium et organisé autour de la notion d'engin. Conformément au projet qui gouverne leur conception, les ouvrages qui procèdent du travail philologique entrepris par Estienne permettent surtout d'entrevoir la manière dont on a pu comprendre le lexique latin de la machina dans la période qui précède les grandes entreprises lexicographiques de l'âge classique. Machina, dans sa teneur morale, tend à se détacher de la forme de tromperie qui était impliquée dans la plupart des représentations lexicographiques médiévales. Le lien entre la machina et la nature pécheresse de l'esprit humain se distend. La latinisation de l'idée permet ainsi de revenir à une conception plus neutre des notions de stratagème et de moyen ingénieux. En retrouvant les éléments cicéroniens par lesquels machina pouvait désigner un mauvais usage de la raison et non une 
raison intrinsèquement corrompue, il est possible d'effacer le clivage que la pensée chrétienne tendait à opérer au sein de la raison, et de revenir à une conception de l'intelligence fertile qui ne serait pas en elle-même porteuse de valeurs moralement dangereuses.

La manière dont les dictionnaires définissent la machina tend, plus généralement, à renforcer continûment le lien qu'elle entretient en amont vers l'esprit dont elle procède, au détriment des aspects techniques et fonctionnels (levage, halage, guerre) qui la définissaient chez Vitruve. L'idée impliquée dans machina possède alors les caractéristiques qui lui confèrent les propriétés d'une articulation, ce qui la rend disponible pour signifier la relation entre la connaissance et l'action. Le terme devient ainsi une sorte de générique au fonctionnement singulier, qui indique moins une catégorie d'objets ou de fonctions qu'un type de rapport entre l'esprit et la matière, une façon qu'a l'esprit d'être investi dans une matière. Aussi, aucune liste des sortes d'objets ou des types de fonctions identifiés comme des machinae (ou comme des machines) n'épuise les possibilités impliquées par le sémantisme de machina (ou de «machine»). Le terme nomme la règle de composition d'un ensemble, celui des pouvoirs dont les machines de levage ou de halage ne sont que des échantillons momentanés. Cette relative indétermination permet à machina, puis à «machine», de se voir investis par les représentations plus ou moins fictives d'une puissance technique que n'absorbent pas les éléments "réels», diversement connus et répertoriés, qui sont nommés, un temps, «machines». Cette dissociation entre l'idée et ses actualisations concrètes et provisoires ouvre la voie qui fait de la machine le lieu des possibles infinis.

Les dictionnaires issus du travail de Robert Estienne ne gardent plus grand chose non plus de la synthèse élaborée par le Moyen Âge entre l'artificialisme platonicien et la notion stoïcienne de Providence. Ainsi, c'est le support théorique des analogies qui permettaient à machina de s'appliquer au monde ou à l'homme, en tant qu'ils sont des créatures divines, qui tend à se distendre lorsque la lexicologie renaissante entreprend la réfection du sémantisme "classique». La sphère de la machina mundi, qui s'affirme cependant avec plus de force dans les éditions du début du XVII ${ }^{e}$ siècle, est représentée presque exclusivement dans les éditions antérieures par l'occurrence lucrétienne, alors que toute référence directe à un "vivant machine» s'efface. Ce retour à l'origine atteste néanmoins la permanence de représentations associées à la machine du monde. Mais il tend à les détacher de leur contexte chrétien pour leur permettre d'exprimer le platonisme renaissant, auquel la référence lucrétienne semble ici servir de caution. Cela ouvre la voie aussi bien à l'appauvrissement ornemental de l'image dont témoignera l'âge classique, qu'à la possibilité de la voir investie par des formes de 
pensée étrangères à l'univers où elle a pris sa force, mais qui, par leur effacement, n'opposent plus guère de résistance.

Déposée dans la langue, l'idée de machina, et celle de machine qui hérite de cette histoire plurielle, se développe désormais selon trois lignes concurrentes qui peuvent s'ignorer ou se croiser. Il y a d'abord une ligne "classique», réformée et humaniste, pour laquelle la machina est un artifice qui relève d'un esprit ingénieux et d'une pratique efficace. On trouve ensuite une ligne théologique et/ou platonisante, pour laquelle machina est l'agencement unifié du divers hétérogène constituant un monde, quelle que soit la manière dont est comprise cette unité, et pour laquelle le terme signifie la composition ordonnée résultant de l'investissement d'un esprit divin infiniment ingénieux dans une matière plus ou moins rebelle. Cette bienveillance ordonnatrice, on peut en percevoir l'efficience localement, dans des phénomènes finalisés de convenances partielles qui permettent d'extrapoler l'ordre du Tout, mais qui ne permettent pas de le comprendre et de le restituer. Dans ce cadre, le tout arrangé qu'est la machine de quelque chose n'a rien de «mécanique», et le grand animal platonicien s'accommode très bien d'être machine en ce sens. Ces deux lignes, enfin, conservent un lien avec l'ancienne mèkhanè, toujours susceptible d'être réactivée lorsqu'il s'agit de faire valoir l'étrangeté intime qu'enveloppe ce qui peut être dit en termes de machina. L'ensemble des données relatives aux significations de machina que procurent les dictionnaires renaissants intéresse directement le lexique français de la machine, qui est alors en cours de formation. Le mot «machine», obtenu par transposition du latin, se fait peu à peu une place, entre «engin» et «instrument». Il vient ainsi compliquer peu à peu l'ancienne configuration formée par les termes de la machination, qui restent les plus fréquents dans les dictionnaires et la langue du $\mathrm{XVI}^{\mathrm{e}}$ siècle. Une tension s'instaure cependant entre deux niveaux différents : celui où continuent d'être présentes les valeurs éthiques auxquelles le Moyen Age avait attaché tant d'importance, et ce nouveau «machine», où se trouvent investis les éléments issus de la réfection humaniste du sémantisme de machina. Le sens encore très peu caractérisé $\mathrm{du}$ terme le met en effet dans la situation de pouvoir traduire l'ensemble de ces valeurs rénovées. Les idées associées à «machine» seront alors dominées par les représentations antiques qui font l'objet du travail d'Estienne sur le lexique dont il hérite de Calepino. «Machine» comme équivalent de machina en reçoit les caractères : laïcisation, aspect concret, valorisation d'un esprit détaché de toute compromission morale ou religieuse. Les deux ordres, celui de la sphère morale et celui de la sphère technique, pourront désormais entrer dans le jeu complexe des compositions et des oppositions que traduira le lexique de la machine au $\mathrm{XVII}^{e}$ siècle, lequel tendra à réinterpréter les valeurs anciennes de la machina- 
tion, fondées sur la transposition des éléments figurés procurés par le lexique latin, en termes techniques (autour de l'idée de ressort par exemple). Les tensions qui déterminent les métaphores "machiniques» de l'âge classique résultent des relations qu'entretiennent les valeurs déposées dans la langue : oppositions entre les éléments techniques et les valeurs associées à l'unité d'un ordre composé ; conflits entre les aspects éthiques de la machination et les aspects pratiques de l'instrument ; divorce, surtout, entre les différentes formes d'esprit dont la machine est la matérialisation. Ce dernier aspect est sans doute déterminant, c'est de lui que témoigne la concurrence entre les deux filiations étymologiques qui partagent les mots de la famille. La machine, en effet, va voir se dissocier l'intelligence pratique qu'elle suppose d'abord et la raison qui connaît et qui analyse, dont elle pourra illustrer les méthodes dans ce qui deviendra le mécanisme. Ce qui est alors réactivé, c'est l'opposition dont témoignait déjà le texte de Vitruve entre la machine comme invention technique empirique et la machine comme objet d'une mécanique théorique, celle à laquelle les premières décennies du XVII siècle donneront l'importance que l'on sait. Poser ce qui est à connaître comme machine, selon une ambivalence qui sera le propre de certains aspects du sémantisme classique, cela servira alors à rendre nécessaire la découverte et l'exposition des mécanismes qui sont impliqués dans et par cette machine. Cette relation de complémentarité et d'opposition caractérisera les rapports entre les deux séries terminologiques tout au long des $\mathrm{XVII}^{\mathrm{e}}$ et $\mathrm{XVIII}{ }^{\mathrm{e}}$ siècles. Cette première évolution implique la seconde : la résistance de l'idée de machine à sa réduction mécaniste. Le rejet d'une rationalité connaissante au nom d'une raison inventrice saura en effet se servir des singularités propres aux machines «réelles» pour les retourner contre les méthodes que le concept de machine élaboré par le mécanisme a pour fonction d'illustrer. Le conflit entre «machine» et "méchanique», qui structure l'ensemble du discours de la machine à l'âge classique, trouve ainsi ses conditions d'expression dans l'histoire des mots et dans la manière dont cette histoire permet de penser les relations entre l'esprit et la matière, telles que la notion de machine permet de les articuler.

D'une part, la machine peut figurer comme un fragment intelligible d'une matière agencée, dans lequel il est possible de retrouver, par analyse, l'ordre rationnel et systématique des lois qui gouvernent l'organisation d'un monde devenu entièrement soluble dans l'intelligence ; d'autre part, elle peut signifier le surgissement d'une singulière étrangeté, celle d'effets et de puissances irréductibles à l'analyse logique et à l'ordre mathématique. Ou bien elle est le modèle d'un ordre où la matière satisfait aux exigences de la géométrisation et où l'esprit se soumet la diversité d'un univers rendu intelligible, ou bien elle est la 
matérialisation de forces par lesquelles la matière devient un creuset opaque, où gît un dynamisme inaperçu et où prolifèrent les formes neuves. Les configurations nouvelles que sont les machines, lorsqu'elles répondent à un ingenium devenu génie, peuvent illustrer la singulière puissance d'engendrement d'une intelligence essentiellement créatrice et active. Pour prendre la mesure de cette opposition, il faut reconstituer, autant que les dictionnaires de l'âge classique le permettent, le chemin qui conduit de Descartes à Diderot, du Dictionnaire de l'Académie à l'Encyclopédie, de l'aristocratique rejet des techniques à la prolifération encyclopédique des machines qui contribue à faire de la description des arts et métiers le labyrinthe dont Diderot parle si souvent.

La machine classique n'est certainement pas un objet socialement neutre, et les valeurs des mots qui s'y rapportent sont marquées par l'idéologie d'un monde où l'exercice des métiers continue d'évoquer l'infamie. Mais c'est aussi un objet au statut indécis capable de s'anoblir diversement, au service de la guerre, de l'Etat, des spectacles, de la science et de la raison, tout en conservant toujours, quel que soit l'endroit d'où on le considère, la trace d'une compromission, d'un oblique qui menace bien souvent l'ordre qu'on lui assigne fréquemment pour tâche de signifier. La lecture des grands dictionnaires classiques suit une procédure qui fait apparaître les valeurs explicites et sous-jacentes de l'ensemble des termes qui composent la famille de "machine», afin de déterminer les relations complexes qui se nouent entre eux. Il s'agit ensuite de confronter la notion de machine avec celles d'engin et d'instrument, puis d'analyser les attendus et les résonances des articles «machine» et de les confronter, pour les éditions numérisées, avec l'ensemble des occurrences significatives de «machine», depuis les plus singulièrement étrangères à nos représentations jusqu'à celles qui montrent le rôle que joue "machine» dans la connaissance. Ce protocole permet de montrer que l'idée de machine trouve son centre de gravité lorsqu'elle désigne un moyen efficace dont on ignore la nature précise des procédures qui le rendent opérant, dont on ignore, très exactement, le mécanisme. Le mécanisme comme doctrine se fonde sur la réduction de la machine à son mécanisme, et finalement sur la négation de ce que «machine» enveloppe d'obscurité. La machine est ce qui est à connaître, le mécanisme ce qui est connu, ou supposé tel. L'effort cartésien, l'hygiène de l'esprit en quoi consiste la méthode, c'est le moyen très sûr de résorber la machine dans la clarté analytique du mécanisme. En cela, rien n'est plus opposé à la machine que le mécanisme. A la faveur d'un curieux composé d'inconnu, de simplicité et de mystère, la notion de machine va prendre un visage tout à fait étranger à l'érudition humaniste et à la rigueur cartésienne. Cet aspect, les dictionnaires en rendent compte. Mais l'une de ses expressions les plus nettes se trouve sous la 
plume de Charles Sorel, dans un passage de son traité De la connaissance des bons livres ou examen de plusieurs autheurs? ${ }^{7}$.

Si l'on nomme «machine» (et plus tard «machin») ce dont on ignore le nom, c'est parce que l'idée de machine implique celle d'inconnu. Parler de "machine», cela implique qu'il y a dans l'objet quelque chose qui pourrait faire l'objet d'un savoir, mais qui demeure ignoré et qui est, peut-être, irréductible à tout principe clair et distinct. La machine c'est un savoir possible vu du côté de l'ignorance ; le mécanisme, c'est ce même savoir rendu présent. «Machine» et «mécanisme» forment ainsi un couple : le même objet est considéré à partir de deux points de vue différents, opposés et souvent inconciliables. Il s'agit de machine lorsque l'objet est envisagé comme étrange et mystérieux, quand les moyens qui produisent son effet restent indéterminés, quand on ignore les procédures exactes de son efficience, quand l'objet participe de compétences mal partagées. Toutes les nuances de cette indétermination peuvent être convoquées : l'inconnaissable (les ressorts divins de la

7 Charles Sorel, De la connaissance des bons livres ou examen de plusieurs autheurs, 1671. L'édition consultée est celle, suivie des Suppléments des Traités de la connaissance des bons livres, que présente Hervé Béchade (Paris, Slatkine, 1981). Le passage se trouve dans le quatrième traité, Du nouveau Langage François, ou du Langage à la Mode ; Tiré de quelques Livres de nostre Siècle, E principalement des ENTRETIENS D'ARTISTE E $D^{\prime} E U G E N E$. Chap. IV, dans la partie intitulée «De l'utilité des nouveaux mots à la mode», p. 421-422: «Voilà le secret de ce qui concerne nos beaux mots : toute l'estime qu'on fait d'eux ne vient que du profit qu'on en reçoit. Cela est si commode de dire, Je m'en vais me mettre sur un tel Chapitre. Vous vous mettrez sur ce pied-là. J'ay bien la mine de ne rien savoir d'une telle chose ; avec plusieurs autres façons de parler qui vous exemptent d'en chercher de plus significatives. Ceci a le même effet que les Quolibets du peuple ; on peut dire que ce sont les Quolibets galants, ou les Quolibets $\mathrm{du}$ beau Monde, mais on a raison de craindre qu'à la fin ils ne passent au Peuple comme beaucoup d'autres : toutefois on ne s'en sert qu'en tant qu'ils sont en crédit. Les Livres d'Observation sur la langue Françoise, ne nous on pas déclaré cette utilité ; Il suffit que nous la reconnaissions maintenant. Nous voyons que cela nous a fait grand plaisir d'avoir de ces mots à la mode, parce qu'il s'en trouve même qui tous seuls signifient tout ce qu'on veut. Il y a quantité de Gens qui lors qu'ils ne peuvent exprimer quelque chose par un mot propre, usent du mot de Machine, ils disent, Il faut faire des machines pour cela, \& que ce sont là des Machines. Les autres se servent par tout du mot d'Affaire ; Ils signifient par là toutes les choses dont ils ne peuvent trouver le nom : Les femmes en parlant de leurs bijoux \& de leurs façons de s'habiller, diront, On porte à cette heure de certaines affaires. Quelquefois les Hommes emploient aussi le nom d'affaires, \& quelques autres, pour des choses fort dissemblables, soit pour des étoffes, des meubles, des édifices, \& des différentes actions de la vie. Tout cela est Affaire ou Machine». 
création), l'indéterminé, le dissimulé et le secret (les ressorts de la politique), 1 'inconnu qui demande à devenir connu et qui mobilise les efforts de la science. Parler de «machine» peut supposer que l'objet est connaissable (on peut retrouver le mot juste, apprendre la mécanique, démonter la montre), mais que cette connaissance est déléguée à un autre, à un spécialiste, dont on ne s'inquiète guère. Ce spécialiste de la machine, c'est le machiniste ou le mécanicien, qui détiennent un savoir technique ; c'est aussi le savant qui connaît, ou qui est réputé connaître, la mécanique de la nature. En revanche, parler de "mécanique», cela revient à considérer l'objet non seulement comme connaissable et maîtrisable, mais comme connu et maîtrisé. Selon le type de savoir impliqué, la maîtrise sera ou bien considérée comme positive et valorisée ( $c$ 'est en général le cas pour la science), ou bien mineure et dévalorisante, puisque trop facile et seulement technique, comme c'est le cas dans certains emplois «esthétiques» du terme (la mécanique de la poésie). Ainsi, «machine» s'oppose aux deux valeurs de «mécanique», à la compétence technique de l'artisan comme au savoir clair et distinct de la science. La relation entre "machine» et "mécanisme» est assez bien décrite par le sens technique moderne (et souvent déjà classique) de «mécanisme»: celui de la montre, c'est l'ensemble des pièces qui produisent le mouvement réglé et qui donnent son sens à l'objet. Du point de vue fonctionnel il n'y a rien de plus dans la montre que son mécanisme, on peut entièrement l'y réduire. Mais «machine» continue de contenir "mécanisme», comme le tout de la montre contient le mécanisme : le boîtier, la structure extérieure, les parties fixes, etc., forment, avec le mécanisme, la machine qu'est la montre. Cette enveloppe extérieure et dissimulatrice porte le sens de "machine» : elle est nulle pour ce qui regarde la fonction (elle ne produit rien, elle protège, assure la cohésion...), mais surtout elle cache, elle dissimule et elle participe ainsi du mystère d'un objet dont le tout reste irréductible à la somme des parties.

JEAN-LUC MARTINE

Université de Bourgogne 